\title{
Deflection of light and particles by moving gravitational lenses
}

\author{
Olaf Wucknitz ${ }^{*}$ \\ Universität Potsdam, Institut für Physik, Am Neuen Palais 10, 14469 Potsdam, Germany \\ Ulrich Sperhake \\ Pennsylvania State University, Centers for Gravitational Physics 8 Geometry \\ and for Gravitational Wave Physics, University Park, Pennsylvania 16802, USA \\ (Dated: 19 Jan 2004; Received 6 Mar 2003; Revised 2 Sep 2003; Accepted 24 Nov 2003)
}

\begin{abstract}
Various authors have investigated the problem of light deflection by radially moving gravitational lenses, but the results presented so far do not appear to agree on the expected deflection angles. Some publications claim a scaling of deflection angles with $1-v$ to first order in the radial lens velocity $v$, while others obtained a scaling with $1-2 v$. In this paper we generalize the calculations for arbitrary lens velocities and show that the first result is the correct one. We discuss the seeming inconsistency of relativistic light deflection with the classical picture of moving test particles by generalizing the lens effect to test particles of arbitrary velocity, including light as a limiting case. We show that the effect of radial motion of the lens is very different for slowly moving test particles and light and that a critical test particle velocity exists for which the motion of the lens has no effect on the deflection angle to first order. An interesting and not immediately intuitive result is obtained in the limit of a highly relativistic motion of the lens towards the observer, where the deflection angle of light reduces to zero. This phenomenon is elucidated in terms of moving refractive media. Furthermore, we discuss the dragging of inertial frames in the field of a moving lens and the corresponding Lense-Thirring precession, in order to shed more light on the geometrical effects in the surroundings of a moving mass. In a second part we discuss the effect of transversal motion on the observed redshift of lensed sources. We demonstrate how a simple kinematic calculation explains the effects for arbitrary velocities of the lens and test particles. Additionally we include the transversal motion of the source and observer to show that all three velocities can be combined into an effective relative transversal velocity similar to the approach used in microlensing studies.
\end{abstract}

PACS numbers: 95.30.Sf, 04.20.Cv, 04.25.Nx, 98.62.Sb

\section{INTRODUCTION}

The subject of gravitational lenses (light deflection by gravitational fields in the Universe) is a well-established field in modern astrophysical research (see, e.g., 1, 2, 3, 4]). Applications include cosmology, dark matter, the large scale structure in the Universe, clusters of galaxies, galactic structure, the structure of the Milky Way, and even the search for extrasolar planets. Not to be forgotten, light deflection by the Sun's gravitation was the first test of the then new theory of general relativity [5].

In almost all of the studies, the deflecting masses are treated as being at rest in the cosmological RobertsonWalker metric or (in the limit of noncosmological lensing) at rest with respect to the source and observer. This is well justified since most sufficiently massive astronomical lenses have velocities very small compared to the velocity of light, which leads only to minor gravitomagnetic corrections.

In addition to the potential astrophysical relevance, our main motivation to study moving lenses is the desire to understand the fundamental physics governing the light deflection caused by such systems in an intuitive and

\footnotetext{
*Electronic address: olaf@astro.physik.uni-potsdam.de
}

${ }^{\dagger}$ Electronic address: sperhake@astro.psu.edu possibly Newtonian interpretation. This leads us to the comparison of the deflection of light with the deflection of slowly moving particles and especially the effect any motion of the lens has on either.

In contrast to most previous work, we do not only consider effects to first order in velocity (FOV) but for the first time calculate the deflection for arbitrary velocities of both lens and deflected particle (including light in the ultrarelativistic limit).

To our knowledge, the first calculations of the deflection of light in gravitational fields were done by Cavendish (see Will [6]) and Soldner [7]. This early work is based on the ansatz of Newtonian particles moving with the speed of light. Einstein [8] used the principle of equivalence to avoid the physically unsound picture of classical particles for the description of light. The result is naturally the same. Only after the formulation of general relativity the result had to be revised to be twice as large as expected from classical theory [9]. This relativistic result has been confirmed by numerous tests with very high accuracy, see, e.g., 10, 11.

Earlier studies in the context of moving gravitational lenses were undertaken by several authors. In 1993 Pyne and Birkinshaw 12 calculated the effects of the lens' motion on the deflection in a Minkowski background metric. For radial motion with velocity $v$ they found the deflection angle to scale with a factor $1-v$ in FOV approximation. The deflection increases if light and lens are 
moving in opposite directions (as seen by the observer). Interestingly, the leading term is of first order; i.e., the effect is not merely a consequence of scaling with the special-relativistic parameter $\gamma=1 / \sqrt{1-v^{2}}$.

In contrast to this, Capozziello et al. 13] found a different scaling behavior of $1-2 v$ for point-mass lenses. They later generalized their calculations to other mass distributions with the remarkable but questionable result that the scaling of the deflection caused by a rigidly moving lens does not only depend on the mass distribution and velocity of the lens but also on its internal parameters 14]. This is of particular significance because it would enable us to derive information from such observations about the inner structure of lenses beyond their mass and momentum. The same scaling for point mass lenses was found by Sereno 15 and later generalized for other mass distributions in [16].

An alternative analytic method was used by Frittelli, Kling, and Newman [17] which confirmed the result of [12]. Frittelli [18] also discusses the discrepancy between the two results, again favoring [12]. Below we will demonstrate how we find our results to agree with those of 12, 17, 18, 19], whereas our velocity corrections differ by a factor of 2 from the values of $13,14,15,16$.

A further interesting result of 12 concerns the effect of a transversal motion of the lens on the observed redshift of the source. We believe this to be the most promising possibility of actually measuring gravitomagnetic effects in gravitational lensing and even utilizing them for astrophysical studies. Concrete practical scenarios are discussed by Molnar and Birkinshaw [19].

Kopeikin and Schäfer [20] presented an exhaustive calculation of light propagation in the field of an ensemble of arbitrarily moving point masses in terms of retarded Liénard-Wiechert potentials, covering effects on both deflection angles and redshifts.

Aside from gravitational lenses of standard astronomical origin, the deflection of light by topological defects in the universe, such as cosmic strings, has stimulated a great deal of scientific investigation. Of most interest in the context of our work is the study of moving cosmic strings (see, for example, de Laix and Vachaspati 21] and Uzan and Bernardeau 22]). Below we will discuss the similarities and differences arising from such different types of lenses and motions.

This paper is organized as follows. After a summary of the notation and approximations used in this work, we begin our discussion with the analysis of purely radial motion of the gravitational lens in Sec. IIII In view of the fact that the gravitational lensing effect is commonly described in the framework of Newtonian physics, it will be important to verify to what extent this approximation remains valid for moving lenses. For this purpose we will present a Newtonian calculation of the deflection of massive test particles in Sec. IIA and contrast the results with the relativistic expressions derived in Secs. IIIB and IIIC The dependency of the deflection on the test particle's velocity will be studied in detail in Sec. IIID We then turn our attention to the effect of transversal motion of the lens in Sec. [D] and conclude in Sec. $\mathbb{D}$.

A comparison of our analytic results for radial motion with more accurate numerical simulations is presented in Appendix @ An illustration of how some of the calculated effects can be understood in a more intuitive fashion in terms of moving refractive media is given in Appendix B Finally we discuss in Appendix C the dragging of inertial frames in the field of a moving lens by calculating the Lense-Thirring precession in this case.

\section{NOTATION AND APPROXIMATIONS}

In this work we will measure velocities in units of the speed of light, i.e., we set $c=1$. The velocity of the lens is denoted by $v$ and that of the test particle by $w$. The deflection of light is obtained in the limit $w=1$. Without loss of generality we perform all calculations in " $2+1$ " dimensions, described by Cartesian coordinates $y$ and $z$ and time $t$. For all relativistic calculations we will use the metric signature $(+--)$.

Unless stated otherwise we use the weak-field and small-angle approximation as is common in gravitational lens theory. We restrict our discussion to thin lenses so that it is sufficient to calculate the deflection of light in a cosmologically small region around the lens only. We are thus able to describe the surroundings of the lens by the Minkowski metric. The local deflection angle calculated in this way can then be used as part of the cosmological lens equation in which it is related with cosmological distances and the observed positional displacement, see, e.g., [4].

In the weak-field limit, the line element in the rest frame of the lens can be written as

$$
\mathrm{d} s^{2}=(1+2 \Phi) \mathrm{d} t^{\prime 2}-(1-2 \Phi)\left(\mathrm{d} y^{\prime 2}+\mathrm{d} z^{\prime 2}\right) .
$$

Here we have used primed coordinates to distinguish the rest frame of the lens from that of the observer (unprimed coordinates). The static Newtonian gravitational potential $\Phi$ is linear in the mass distribution and satisfies $|\Phi| \ll 1$

The unperturbed light/particle is traveling in the positive $z$ direction at $y=0$ with velocity $w$ as measured in the unprimed observer system. The deflected trajectories will therefore be directed at small angles relative to the $z$ axis. The only requirement we impose on the velocity of the test particle is that it travels faster than the lens, i.e., $w>v$, so that it passes the lens in the usual direction.

The approximations imply that deviations of $y$ from the unperturbed path $y \equiv 0$ need to be calculated to first order only. This "first order in deflection" (FOD) approximation is not to be confused with FOV. Below, these approximations will allow us to integrate along the unperturbed path instead of the deflected (and then still unknown) path itself. We will further be able to compare the deflection of light for lenses at different velocities without changing the light path close to the lens, 
i.e., without changing the impact parameter. In this approximation we can also relate the coordinates along the path of the test particle by

$$
\mathrm{d} z=w \mathrm{~d} t
$$

The deflection angle is the difference of the propagation directions before and after passing the lens. For small angles and propagation close to the $z$ direction, this can be written as

$$
\alpha=\left.\frac{\mathrm{d} y}{\mathrm{~d} z}\right|_{\text {out }}-\left.\frac{\mathrm{d} y}{\mathrm{~d} z}\right|_{\text {in }} .
$$

For the ingoing path we have $z \rightarrow-\infty$, for the outgoing $z \rightarrow+\infty$.

For the radial velocity of the lens and the test particle we will adopt the sign-convention that they are positive if directed towards the observer so that $v$ and $w$ are measured in the same direction. This definition is opposite to the usual astronomical definition of radial velocities but is commonly used in the literature on moving gravitational lenses.

\section{RADIAL MOTION}

\section{A. Nonrelativistic calculation based on the principle of equivalence}

We begin our discussion of the effect of purely radial motion of the lens on the resulting deflection angle with a Newtonian discussion. Aside from providing an intuitive insight into the effect, the results thus obtained will also enable us below to highlight the quantitative and qualitative modifications arising in a general relativistic treatment.

In order to avoid a description of light in terms of classical particles, we follow the lines of Einstein [8] and apply the principle of equivalence to the gravitational effects and use nonrelativistic kinematics otherwise. At each point of the path of a test particle, we can define a freely falling observer who is momentarily at rest and who views the deflected path in her vicinity as a straight line. The equations of motion of this observer are given by

$$
\frac{\mathrm{d}^{2} y}{\mathrm{~d} t^{2}}=-\Phi_{y}, \quad \frac{\mathrm{d}^{2} z}{\mathrm{~d} t^{2}}=-\Phi_{z}
$$

where $\Phi_{y}$ and $\Phi_{z}$ denote partial derivatives of the potential with respect to $y$ and $z$, respectively. In the frame of this observer, the acceleration of the test particles vanishes, so that they follow the same equations of motion (4) as the falling observer.

In order to calculate the deflection angle defined in Eq. (3) we need to calculate $\mathrm{d} y / \mathrm{d} z$. In FOD this is obtained from Eq. (4) by replacing time derivatives with $z$ derivatives according to Eq. (2) and integrating once over $z$. We thus obtain for the deflection angle

$$
\alpha_{w}^{\mathrm{cl}}(v)=-\frac{1}{w^{2}} \int_{-\infty}^{\infty} \mathrm{d} z \Phi_{y}(y, z) .
$$

Next we transform the integral to primed coordinates. First we note that the boost does not affect the $y$ direction, so that $\Phi_{y}(y, z)=\Phi_{y^{\prime}}\left(y^{\prime}, z^{\prime}\right)$. Secondly we can use Eq. (2) together with the nonrelativistic limit of the Lorentz transformation (11) below to obtain $\mathrm{d} z^{\prime}=$ $(1-v / w) \mathrm{d} z$. This gives us the deflection angle as

$$
\alpha_{w}^{\mathrm{cl}}(v)=-\frac{1}{w(w-v)} \int_{-\infty}^{\infty} \mathrm{d} z^{\prime} \Phi_{y^{\prime}}\left(y^{\prime}, z^{\prime}\right) .
$$

The integral is now expressed entirely in terms of the coordinates of the lens' rest frame and is therefore independent of the velocity $v$ (the perturbation of the gradient $\Phi_{y^{\prime}}$ on a perturbed path is of second order in the deflection angle and is thus ignored in FOD). We can therefore express the dependency of the deflection angle on the velocity $v$ of the lens in FOV approximation as

$$
\begin{aligned}
& \frac{\alpha_{w}^{\mathrm{cl}}(v)}{\alpha_{w}^{\mathrm{cl}}(0)}=\frac{w}{w-v} \\
& \alpha_{w}^{\mathrm{cl}}(0)=-\frac{1}{w^{2}} \int_{-\infty}^{\infty} \mathrm{d} z^{\prime} \Phi_{y^{\prime}} .
\end{aligned}
$$

The factor $1 / w^{2}$ in the last equation can be interpreted as follows. One factor $1 / w$ originates from Eqs. (2) and (3) and represents the geometrical effect that the transversal acceleration due to the gradient $\Phi_{y^{\prime}}$ and the resulting change in velocity along the $y$ direction $\Delta w_{\perp}$ correspond to a larger deflection angle the smaller the velocitycomponent $w$ along the $z$ direction. The second factor $1 / w$ is due to the interaction time which scales inversely with the velocity $w$. The first contribution is unchanged for moving lenses while the interaction time now scales inversely with the relative velocity $w-v$, leading to the correction factor in Eq. (77).

This result is not only exact in the nonrelativistic limit $(w \rightarrow 0)$ but still provides a decent approximation for the deflection angle of light for lenses at rest: The only modification caused by general relativity is an additional factor of 2. One may ask whether this remains true for moving lenses - can one simply apply the missing scaling factor of 2 to the deflection angle of light in the moving case as well? Below we will learn that this is not the case.

As a qualitative result for slowly moving particles, we find from Eq. (7) that the deflection increases (due to the change in interaction time) if the test particle and lens are moving in the same direction. We will see that the opposite holds for light, so there exists a test particle velocity $w_{\mathrm{c}}$ for which motion of the lens has no effect on the deflection angle in FOV approximation. We will study this feature in more detail in the next sections when we calculate the deflection in the framework of general relativity. 


\section{B. Relativistic calculation in the observer frame}

In order to calculate the trajectories of test particles in the observer's rest frame, in which the lens is moving, we need to find the line element in this system. For this purpose we transform the line element (1) from the rest frame of the lens (primed) to that of the observer (unprimed coordinates). The corresponding Lorentz transformation is given by

$$
\begin{aligned}
\mathrm{d} t^{\prime} & =\gamma(\mathrm{d} t-v \mathrm{~d} z) \\
\mathrm{d} y^{\prime} & =\mathrm{d} y \\
\mathrm{~d} z^{\prime} & =\gamma(\mathrm{d} z-v \mathrm{~d} t)
\end{aligned}
$$

where $\gamma=1 / \sqrt{1-v^{2}}$. We drop the FOV approximation for this calculation so that the line element for arbitrary velocity $v$ in the observer's rest frame is given by

$$
\begin{aligned}
\mathrm{d} s^{2}= & {\left[1+2\left(1+v^{2}\right) \gamma^{2} \Phi\right] \mathrm{d} t^{2}-(1-2 \Phi) \mathrm{d} y^{2} } \\
& -\left[1-2\left(1+v^{2}\right) \gamma^{2} \Phi\right] \mathrm{d} z^{2}-8 v \gamma^{2} \Phi \mathrm{d} t \mathrm{~d} z
\end{aligned}
$$

In the limit of small $v$ this metric reduces to the FOV result of Schneider et al. [4].

The geodesic equation with a given metric is equivalent to an Euler-Lagrange system of equations with Lagrange function

$$
L=\left(\frac{\mathrm{d} s}{\mathrm{~d} \lambda}\right)^{2}
$$

where $\lambda$ is an affine parameter of the test particle's world line. We denote the derivative with respect to this parameter by a dot, e.g., $\mathrm{d} y / \mathrm{d} \lambda=\dot{y}$. Using the line element (12), the equation for $y$ in FOD approximation becomes

$$
\begin{gathered}
\frac{\mathrm{d}}{\mathrm{d} \lambda} \frac{\partial L}{\partial \dot{y}}-\frac{\partial L}{\partial y}=0 \\
\ddot{y}=-\left[\left(1+v^{2}\right)\left(\dot{t}^{2}+\dot{z}^{2}\right)-4 v \dot{t} \dot{z}\right] \gamma^{2} \Phi_{y} .
\end{gathered}
$$

Terms like $\dot{\Phi} \dot{y}, \Phi \ddot{y}$ and $\dot{y}^{2}$ are neglected here because they are of higher order in the deflection.

From the corresponding equations for $z$ and $t$ we learn that deviations from the undeflected path in these coordinates are of the same order as the deflection in $y$ so that they become insignificant after multiplication with $\Phi_{y}$ in Eq. (15). We can therefore apply $\dot{t}=\dot{z} / w$ from Eq. (2) for the undeflected path and use $\lambda=z$ as the affine parameter. Equation (15) can then be written as

$$
\frac{\mathrm{d}^{2} y}{\mathrm{~d} z^{2}}=-\left[\left(1+v^{2}\right)\left(1+w^{-2}\right)-4 \frac{v}{w}\right] \gamma^{2} \Phi_{y} .
$$

After integration we can use Eq. (3) and find the deflection angle

$$
\begin{aligned}
\alpha_{w}(v) & =\int_{-\infty}^{\infty} \mathrm{d} z \frac{\mathrm{d}^{2} y}{\mathrm{~d} z^{2}} \\
& =\frac{1}{(1-v / w) \gamma} \int_{-\infty}^{\infty} \mathrm{d} z^{\prime} \frac{\mathrm{d}^{2} y}{\mathrm{~d} z^{2}}
\end{aligned}
$$

In the final step we applied a parameter transformation from $z$ to $z^{\prime}$ using the unperturbed particle path (2) and the Lorentz transformation (11). This result can again be written as a correction to the deflection angle $\alpha_{w}(0)$ caused by a lens at rest, so that we obtain

$$
\begin{aligned}
& \frac{\alpha_{w}(v)}{\alpha_{w}(0)}=\frac{\gamma}{1-v / w}\left(1+v^{2}-\frac{4 v w}{1+w^{2}}\right) \\
& \alpha_{w}(0)=-\left(1+w^{-2}\right) \int_{-\infty}^{\infty} \mathrm{d} z^{\prime} \Phi_{y^{\prime}}
\end{aligned}
$$

As before the integral in Eq. (20) is evaluated to FOD by using the unperturbed path. We emphasize that in this approximation the impact parameter, and thus the integral in Eq. (20), is a background quantity, i.e. remains constant for different values of $v$ and $w$. In all our comparisons of deflection angles obtained for different velocities we will therefore consider the impact parameter as being kept constant, even in the numerical calculations below which go beyond FOD.

For the special case of light and a lens at rest $(w=1$, $v=0$ ), the comparison of Eq. (20) with the classical result (8) yields the additional factor of 2 introduced by general relativity. In the nonrelativistic limit $|v|, w \ll 1$ we recover the classical result (17).

\section{Relativistic calculation in the lens frame}

In view of the discrepancy between the results presented in the literature (see, e.g., [12] and [13]), we consider it appropriate to calculate the same effect again in the lens' reference frame.

For this purpose we consider Eq. (20) which remains valid in the rest frame of the lens even for nonzero $v$ and which can be derived directly from the usual static weak-field metric (1).

We must take into account in that case, however, that the particle velocity now is $w^{\prime}$ (instead of $w$ ) and that the resulting deflection angle will be $\alpha^{\prime}$, i.e., the angle as viewed in the lens' system. In order to facilitate a comparison with the results of the previous section we need to Lorentz-transform both quantities into the observer's rest frame. We first consider the velocity $w^{\prime}$.

Because of the FOD approximation we can consider the velocities $w, w^{\prime}$ as the components along the $z$ axis for this transformation. This is reflected by Eq. (2) and its analogue in primed coordinates $\mathrm{d} z^{\prime}=w^{\prime} \mathrm{d} t^{\prime}$. Combining these relations with the Lorentz transformation (9)-(11) we directly obtain the relativistic addition of velocities

$$
w^{\prime}=\frac{w-v}{1-v w}
$$

We simplify the transformation of the deflection angle $\alpha$ by defining the direction of the ingoing path as that of zero deflection. We therefore need to transform the outgoing path only which is parametrized by

$$
\mathrm{d} z^{\prime}=w^{\prime} \mathrm{d} t^{\prime}
$$



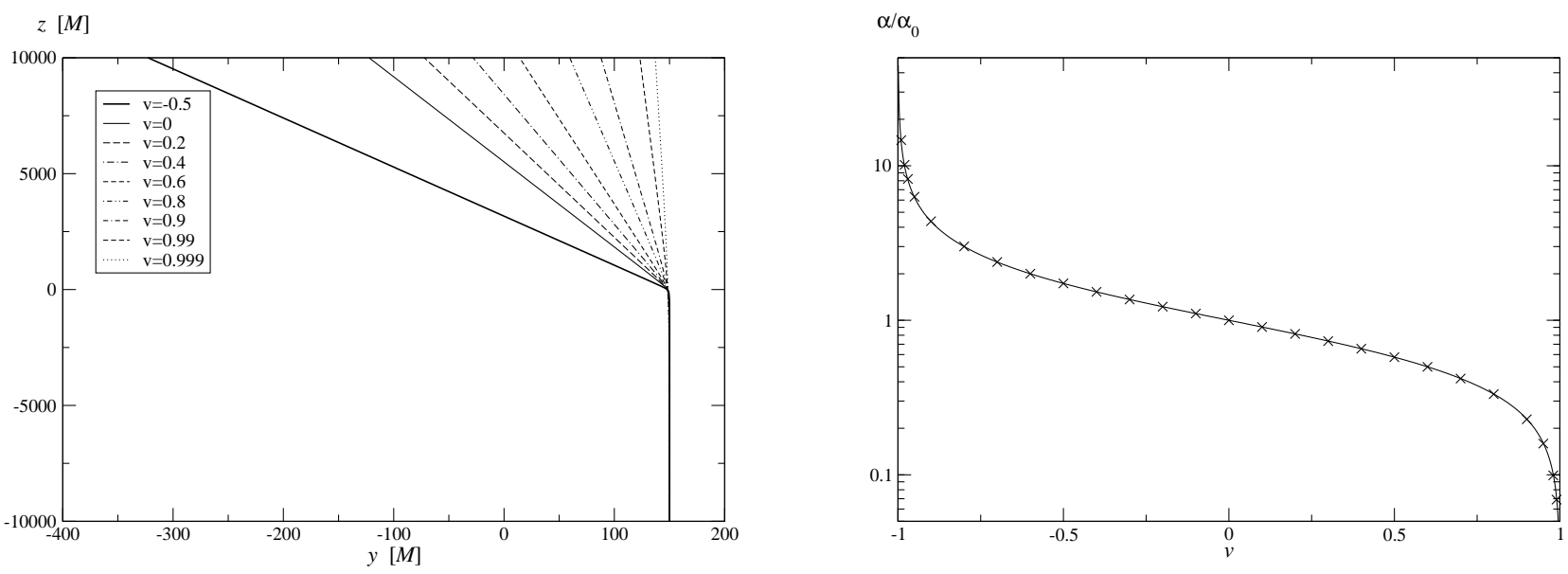

FIG. 1: The null-geodesics (left panel) and the (logarithmic) deflection angle (right panel) are plotted for various velocities of the lens. In the right panel the crosses $(x)$ are the numerically calculated deflection angles, the solid curve is given by the analytic result (26) obtained in FOD approximation. Even though these deflection angles are larger than typical astronomical values by several orders of magnitude (for this calculation $\alpha_{0}=0.027$ ), the FOD approximation still provides excellent results. Following the arguments in Sec. IIIC it can be shown that, even for $v$ quite close to -1 , the relative error (due to the FOD approximation) of $\alpha(v)$ as defined in Eq. (3) is the same as that of $\alpha(0)$ even though the small-angle approximation would not hold for $\alpha(v)$ directly.

$$
\mathrm{d} y^{\prime}=\alpha_{w^{\prime}}^{\prime}(0) \mathrm{d} z^{\prime}
$$

[cf. Eqs. (2) and (3)]. Again we use the Lorentz transformation (9) - (11) to eliminate the primed coordinate differentials and Eq. (3) to express the result in terms of the deflection angle in the observer's rest frame. We thus obtain

$$
\frac{\alpha_{w}(v)}{\alpha_{w^{\prime}}^{\prime}(0)}=\left(1-\frac{v}{w}\right) \gamma
$$

Eventually we want to compare deflection angles for equal test particle velocities $w$, so that we still need to express $\alpha_{w^{\prime}}^{\prime}(0)$ in terms of $\alpha_{w}(0)$. From Eq. (20) we directly obtain

$$
\begin{aligned}
\frac{\alpha_{w^{\prime}}^{\prime}(0)}{\alpha_{w}(0)} & =\frac{1+w^{\prime-2}}{1+w^{-2}} \\
& =\left(1-\frac{v}{w}\right)^{-2}\left(1+v^{2}-\frac{4 v w}{1+w^{2}}\right)
\end{aligned}
$$

which becomes unity in the special case of light $(w=$ $\left.w^{\prime}=1\right)$. The combination of Eqs. (24) and (25) confirms the above result (19) calculated directly from the viewpoint of the observer.

\section{Discussion of radial motion}

\section{Rigidly moving lenses}

We now turn our attention to the interpretation of the deflection of light for a rigidly moving gravitational lens to FOD. For this purpose we consider the limit of Eq. (19) for $w \rightarrow 1$. In that case the scaling has the simple form

$$
\frac{\alpha(v)}{\alpha(0)}=(1-v) \gamma=\sqrt{\frac{1-v}{1+v}} .
$$

To FOV this becomes $1-v$ which confirms the result of [12. while it differs by a factor of 2 from that given in 13. We further note that the quotient $\alpha(v) / \alpha(0)$ diverges for $v \rightarrow-1$ but vanishes in the limit $v \rightarrow+1$. Thus, rather surprisingly, a lens approaching the observer with a highly relativistic velocity will not deflect the passing light at all even though the effective mass-energy of the lens as viewed by the observer diverges in this limit.

In view of the counterintuitive nature of this result one may ask whether the approximation of small deflection angles (FOD) underlying this calculation is still justified in the limit of $v \rightarrow 1$. In order to clarify this point we have numerically solved the full geodesic equations, e.g., Eq. (14), for the case of a point mass $(\Phi=-M / r)$ without FOD approximation. Instead of Eq. (15) we obtain in this case a system of three nontrivial second order ordinary differential equations for $t, y$, and $z$. These equations together with more details of the numerical treatment are listed in Appendix A Note that the only approximation in this numerical calculation is the assumption of a weak gravitational field in the rest frame of the lens. In Fig. 1 we plot the resulting geodesics and the deflection angles obtained for different velocities of the lens together with the analytic result in FOD approximation as given by Eq. (26). For these calculations source and observer are located at $z= \pm 10000 M$, respectively, while the impact parameter, which equals the $y$ position of the source with high accuracy, is $150 \mathrm{M}$. The precise values of these parameters do not affect our results. The 


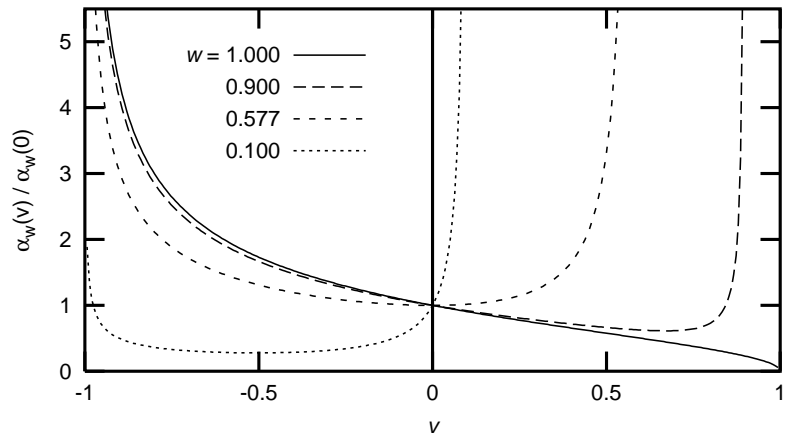

FIG. 2: The deflection angle caused by a moving lens in units of that arising from a lens at rest as given by Eq. (19) is shown as a function of $v$. The graphs are obtained for $w=1$ (light), $0.9,0.577=1 / \sqrt{3}\left(=w_{\mathrm{c}}\right)$, and 0.1. We note that the curve for $w=w_{\mathrm{c}}$ is stationary at $v=0$, i.e., small lens velocities have no effect for this critical test particle velocity.

lens is always positioned at $y=0$ and moves along the $z$ axis, so that it reaches $z=0$ at the same time as the null-geodesics. Note that the $y$ position of the observer depends on the deflection angle since the impact parameter is kept constant as the lens' velocity $v$ is varied.

The excellent agreement between the numerical deflection angle and that predicted by the approximate FOD result demonstrates the validity of the FOD approximation in our calculations even for strongly relativistic velocities $v$.

With regard to the deflection of light by cosmic strings we first note that such types of lenses induce vector and tensor perturbations of the metric in addition to the scalar perturbations in our Eq. (1) [cf. Eq. (38) of Uzan and Bernardeau [22]] which can be combined into an effective deflecting potential. The deflection angle can be expressed in terms of this deflection potential and our result [Eqs. (16), (17)] corresponds to the limit of their Eq. (45) for purely scalar perturbations and linear motion of the lens. In general, 22] find the vector and tensor perturbations to be neglegible as long as the thin lens approximation is valid but not necessarily for the case of extended lenses. A time dependence of the deflecting potential which is of particular interest for string type lenses (because they are expected to move with relativistic velocities) is that of an oscillating loop. For this scenario the deflection of light passing sufficiently far outside the loop has been found to be identical to that of a point mass source (see 21, 22] for details). In contrast, the linear motion studied in this work gives rise to a vector perturbation of the metric, which manifests itself in the $\mathrm{d} t \mathrm{~d} z$ cross term of the boosted line element (12). The analogy between this term and rotational effects will be studied in more detail in Appendix[C]

It remains therefore to obtain a better understanding of how the deflection vanishes in the limit of a lens moving towards the observer at highly relativistic velocities. For this purpose we provide in Appendixes $\mathrm{B}$ and $\mathrm{C}$ a

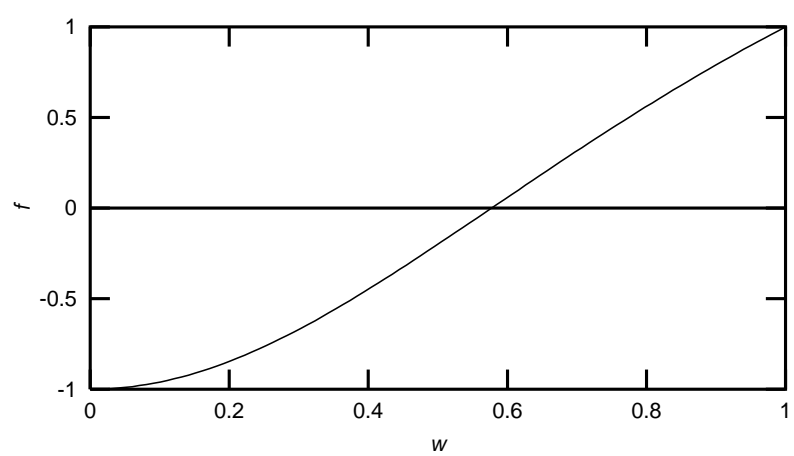

FIG. 3: Effect of small lens velocities $v$ on the deflection angle. According to Eq. (27) the deflection scales with $1-(v / w) f$, where $f=\left(3 w^{2}-1\right) /\left(1+w^{2}\right)$. The graph shows $f$ as a function of the test particle velocity $w$. The effect has opposite signs for $w \rightarrow 0$ and $w=1$ and there is a critical particle velocity $w_{\mathrm{c}}=1 / \sqrt{3}$ for which the lens motion has no effect to FOV.

description in terms of refractive media and the dragging of inertial frames similar to the frame dragging close to rotating bodies.

We now return to the general behavior of the deflection angle for arbitrary velocities $v$ and $w$ which is shown in Fig. 22 This graphic demonstrates the singular behavior of the deflection angle at $v=-1$ and (provided $w \neq 1$ ) $v=w$. The singularity at -1 arises from the diverging factor $\gamma$ while the second singularity is a consequence of the vanishing relative velocities which causes the interaction time to become infinitely large.

We next discuss the limit of small lens velocities $v$ (FOV) combined with a comparatively larger, but otherwise arbitrary speed of the test particle, i.e., $w \gg v$. In this case the scaling of Eq. (19) simplifies to

$$
\frac{\alpha_{w}(v)}{\alpha_{w}(0)}=1-\frac{3 w^{2}-1}{1+w^{2}} \frac{v}{w} .
$$

We have already mentioned above that the deflection of light scales with $1-v$ to FOV whereas the Newtonian result of Eq. (7) predicts a scaling of $1+v / w$ for nonrelativistic test particles satisfying $w \gg v$. Hence there must be a critical velocity $w_{\mathrm{c}}$ of the test particle for which the deflection angle is independent of the lens' velocity $v$ to FOV. From Eq. 27) we see that this critical particle velocity is $w_{\mathrm{c}}=1 / \sqrt{3}$. We illustrate the functional behavior of Eq. (27) in Fig. 3

Only after finishing our calculations we learned that special properties of the velocity $1 / \sqrt{3}$ have been discussed before by Carmeli [ 223] and problem 5.5.1 in [24]]. There it was found that the coordinate velocity of a massive particle approaching the center of a Schwarzschild metric in a radial direction increases only for asymptotic starting velocities smaller than $1 / \sqrt{3}$ but decreases otherwise, all in a FOD approximation. The same was later independently rediscovered by Blinnikov, Okun, and Vysotsky 25, 26]. It can indeed be shown easily that the same is true for any $1+1$ dimensional weak field 
with a metric of $\mathrm{d} s^{2}=(1+2 \Phi) \mathrm{d} t^{2}-(1-2 \Phi) \mathrm{d} r^{2}$. To first order in $\Phi$, there is no coordinate acceleration for $w=w_{\mathrm{c}}$. This effect can in principle be detected by measuring round trip travel times.

With regard to the observation of the deflection caused by a radially moving lens we note that it is not possible to distinguish the scaling of the deflection angle with $\alpha_{w}(v) / \alpha_{w}(0)$ from a scaling with the total mass of the lens. For a given test particle, the effect can therefore be used to infer a radial motion of the lens only if an accurate and independent estimate of the lens' mass is available. An alternative way to disentangle the two effects would consist of measuring deflection angles of test particles with different velocities $w$. Unfortunately, appropriate test particles other than light are currently not available in astrophysics. Even if we could measure the direction of cosmic rays consisting of massive particles with higher accuracy than is possible with existing instruments, they would not serve our purpose as they travel at highly relativistic speeds and would be subject to practically the same deflection as light. Furthermore they are also deflected by electric and magnetic fields.

\section{Compound lenses}

It is an interesting question whether the gravitomagnetic correction to the deflection of light or particles could be used to deduce information about the inner structure (e.g., the velocity field) of a lens as suggested by the results of Capozziello and Re 14 for the deflection of light. We address this question by considering a compact (relative to the impact parameter $r$ ) lens which we treat as a collection of point masses located close to each other. In terms of our formalism, the argument in favor of the possibility to probe the inner structure of the lens can be summarized as follows. Consider a lens with two components of equal mass $m$ moving in opposite directions with the same speed. Compared to a lens at rest with mass $2 m$, we expect a deflection angle larger by a factor $\gamma$ because the compound lens will appear as a lens of mass $2 m \gamma$ in the observer's rest frame. Because of the more complicated dependence of its right-hand side on $v$, however, Eq. (19) will predict a different deflection angle for this scenario. It appears therefore that the deflection is not uniquely determined by the total four-momentum of the lens.

This argument is not valid, however, because the compound lens must be compact not only momentarily but for the whole interval during which the test particle passes by and is deflected significantly. Otherwise the lens would effectively be extended so that the deflection would test rather the mass distribution than the internal velocity field of the lens. Measurable effects are not surprising in that case.

The time scale for the passage is $r / w$ and the displacements of the lens' components during this interval are $r v / w$. Because these displacements must be $\ll r$ to pre- serve the compactness of the lens, we obtain an upper limit for the velocities of its components, $|v| \ll w$. In this limit, however, the right-hand side of Eq. (19) becomes approximately $\gamma(1+$ const $v)$ and the total deflection is indeed determined by the total four-momentum. We thus conclude that no information about the internal structure beyond the mass and total momentum of a compact lens can be obtained from the lensing effect. In the case of light $(w \rightarrow 1)$ this is also demonstrated by Eq. (26), where the deflection is always determined by the total four-momentum, $\alpha \propto p_{t}-p_{z}=\sum_{j}\left(1-v_{j}\right) \gamma_{j} m_{j}$.

\section{E. Comparison with previous results}

We will now address the question of what causes the discrepancies between our results and those in [13, 15] where the velocity effects appear to be overestimated by a factor of 2. Note that this also affects the ensuing results published in [14, 16] which generalize to mass distributions other than point masses. Both these groups exclusively use light as test particles, work in a FOV approximation and use essentially the same approach as Schneider et al. [4] in formulating the problem. ${ }^{1}$ Following this approach, we use the equivalent formulation of light propagation in an Euclidean metric with an effective index of refraction $n$ (cf. Appendix B). For a rigidly moving lens with gravitational potential $\Phi$, this index can be calculated from setting $\theta=0$ in Eqs. (B2) and (B3) and is given by

$$
n-1=-2(1-2 v) \Phi
$$

which does indeed contain the factor $1-2 v$. In order to obtain the correct scaling, however, we must further take into account that the motion of the lens affects the potential $\Phi$ which now becomes time-dependent in the observer's rest frame. It is most convenient to calculate the total deflection integral over the path of light in the primed coordinates of the lens' rest frame. We thus replace $\Phi(t, y, z)=\Phi\left(y^{\prime}, z^{\prime}\right)$ with $z^{\prime}=z-v t$ from Eq. (11) and substitute $z^{\prime}$ for $z$ in the integral. The same procedure has been used in Eq. (7) for the classical and in Eq. (18) for the relativistic calculation. This correction describes the scaling of time of interaction caused by the different relative velocity. The resulting additional factor $1+v$ was not applied in [13] and 15] but would otherwise have changed their scaling factor $1-2 v$ to the correct result of a $1-v$ scaling of light deflection in FOV approximation. Frittelli [18], on the other hand, did apply this factor in her Eqs. (33) and (34) and arrives at the same result as we do in Secs. आIB and ПIC above.

\footnotetext{
${ }^{1}$ An alternative derivation based on integration of the geodesic equation is presented additionally in [13]. Our arguments are valid for this approach as well.
} 


\section{TRANSVERSAL MOTION}

\section{A. Deflection angle}

Until now we have restricted our discussion to a radial motion of the lens, because this velocity component is the only one affecting the deflection angle to FOV. In this section we will discuss the effects of transversal motion. For this purpose we use the same approximations as in the discussion of radial velocity effects, i.e. we allow for weak deflections only and work in the FOD approximation. For radially moving lenses, the light path across the lens does not depend on the velocity so that a nonzero velocity merely gives rise to an overall scaling factor. This is no longer the case for large transversal velocities. Due to aberration, the direction of light propagation in the lens' rest frame does depend on the velocity so that an additional term involving the radial derivative $\Phi_{z^{\prime}}$ appears in the geodesic equation. The resulting scaling law would therefore depend on the mass distribution and is not of general validity and immediate interest.

By using the methods of Secs. IIIB and IIC however, it can be shown that to FOV the deflection angle is not modified at all by transversal motion.

\section{B. Redshift}

Even though a transversal motion of the lens does not affect the deflection angle to FOV, there is an interesting effect on the observed redshift of the source. This can be illustrated as follows. When the test particle approaches the lens it falls into the potential well and gains energy. During the passage, the lens is moving, so that the potential experienced by the particle as it climbs out of the potential well will differ from that before. The associated loss in energy of the test particle will therefore not exactly compensate for its earlier gain. The resulting net change in energy can be measured as a change in the velocity of the test particle or the energy, i.e., wavelength, in the case of a photon.

Alternatively one can view the effect in the following way. The test particle traveling on the rear side of the lens (with respect to the transversal motion) will be deflected towards the lens and slow down the motion of the lens so as to preserve total momentum. The energy loss of the lens (which is proportional to its initial velocity, see below) has to be transferred to the particle. For particles passing on the other side of the lens, this effect has the opposite sign. We will now quantify these ideas straightforwardly by calculating the effect in the framework of special relativistic kinematics.

The transversal momentum transferred from the lens to the test particle in the course of the deflection is given by

$$
\Delta p_{\perp}=\alpha p_{\|}
$$

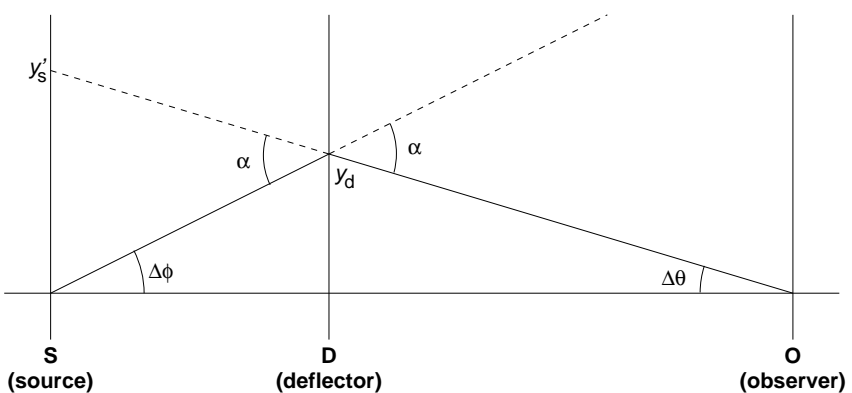

FIG. 4: The lensing geometry. The solid line shows the deflected light path while the horizontal axis is the undeflected light path from the source to the observer. The deflection angle is $\alpha$ and the apparent position of the source is $\Delta \theta$. The true position would be at an angle of zero. As shown in the figure, $\alpha$ is negative while $\Delta \theta, \Delta \phi$, and $y_{\mathrm{d}}, y_{\mathrm{s}}^{\prime}$ are positive.

where $p_{\|}$is the radial momentum of the particle. We can calculate the accompanying transfer of energy $\Delta E$ by using the fundamental equations of special-relativistic kinematics

$$
E^{2}=m^{2}+p^{2}, \quad \mathbf{p}=E \mathbf{v}, \quad E=m \gamma .
$$

From the first of these equations we derive $E \Delta E=\mathbf{p} \cdot \Delta \mathbf{p}$ to first order in $\Delta E$ and thus, together with the second one,

$$
\Delta E=\mathbf{v} \cdot \Delta \mathbf{p} .
$$

We now apply this equation both to the lens and the test particle to write the equation of energy conservation as

$$
v_{\|} \Delta p_{\|}+v_{\perp} \Delta p_{\perp}=w \Delta p_{\|} .
$$

The left-hand side is the energy loss of the lens, written in terms of a possible radial velocity component of the lens $v_{\|}$and its transversal velocity $v_{\perp}$. On the right-hand side we have the energy gain of the test particle. Here the transversal part is of higher order in the deflection and can be neglected. Combining Eqs. (29) and (32) we directly obtain the relative change in radial momentum of the particle

$$
\frac{\Delta p_{\|}}{p_{\|}}=\alpha \frac{v_{\perp}}{w-v_{\|}} .
$$

Even though this relation is valid for arbitrary values of the velocities, we will assume $v_{\|}=0$ for the following discussion.

In the case of light and assuming $v_{\|}=0$ we can relate the relative change in momentum to a shift in frequency or an additional redshift of $\Delta z=-\alpha v_{\perp}$, which agrees with the FOV results of Pyne and Birkinshaw 12] and Kopeikin and Schäfer 20]. In spite of the remarkably simple derivation, however, our results remain valid for arbitrary lens potentials and lens and particle velocities. Equations (29) - (33) do not even depend on the physical process which causes the deflection but instead are valid for any kind of scattering by a massive moving target. 


\section{Geometry}

So far we have only considered a transversal motion of the lens but assumed that source and observer are at rest. In reality, source, lens, and observer will all be moving relative to the cosmological Robertson-Walker background metric. It is therefore necessary to also take into account the motion of source and observer. We will calculate the resulting total redshift in the framework of the FOV approximation. The fundamental geometry is shown in Fig. 4. If the source is moving with a transversal velocity $v_{\mathrm{s}}$, this will lead to a velocity component in the direction of the real path of light and, thus, a Doppler velocity of $v_{\mathrm{s}} \Delta \phi$. We have already seen in the discussion of Eq. (33) that a transverse velocity $v_{\mathrm{d}}$ of the lens leads to a Doppler shift analogous to an apparent radial velocity $v_{\mathrm{d}} \alpha$ of the source. The motion of the observer themself gives rise to an additional contribution corresponding to a radial velocity of $v_{\mathrm{o}} \Delta \theta$. In FOV approximation, the net Doppler effect is obtained by linear addition of these velocities because $(1+\Delta z)$ acts multiplicatively, see Eq. (38) below.

If we denote the apparent size distance parameter ${ }^{2}$ from $a$ to $b$ (angle measured at $a$, size measured at $b$ ) with $D_{a b}$, we can use two equations each to express the apparent position of the source $y_{\mathrm{s}}^{\prime}$ and the position of the lens $y_{\mathrm{d}}$ using different angles and distances (cf. Fig. 4

$$
\begin{aligned}
& y_{\mathrm{s}}^{\prime}=-\alpha D_{\mathrm{ds}}=\Delta \theta D_{\mathrm{os}}, \\
& y_{\mathrm{d}}=\Delta \theta D_{\mathrm{od}}=\Delta \phi D_{\mathrm{sd}} .
\end{aligned}
$$

These equations can be used to express $\alpha$ and $\Delta \phi$ in terms of the apparent displacement $\Delta \theta$ (also called apparent deflection angle) to write the total Doppler velocity in terms of this angle. Because of the expansion of the Universe, distance parameters depend on the direction, i.e., $D_{\mathrm{sd}} \neq D_{\mathrm{ds}}$. The two can easily be related, however, using the redshifts $z_{\mathrm{s}}$ and $z_{\mathrm{d}}$ of source and lens so that we obtain

$$
\frac{D_{\mathrm{sd}}}{D_{\mathrm{ds}}}=\frac{1+z_{\mathrm{s}}}{1+z_{\mathrm{d}}} .
$$

This leads to the final expression for the total Doppler velocity

$$
v_{\text {Dopp }}=\left(\frac{1+z_{\mathrm{d}}}{1+z_{\mathrm{s}}} \frac{D_{\mathrm{od}}}{D_{\mathrm{ds}}} v_{\mathrm{s}}-\frac{D_{\mathrm{os}}}{D_{\mathrm{ds}}} v_{\mathrm{d}}+v_{\mathrm{o}}\right) \Delta \theta .
$$

Remember that our sign convention for $v_{\text {Dopp }}$ is opposite to that commonly used for astronomical radial velocities, so that the observed redshift $z_{\mathrm{s}}^{\prime}$ is given as a modification of the unperturbed redshift $z_{\mathrm{s}}$ in the following way:

$$
1+z_{\mathrm{s}}^{\prime}=\left(1+z_{\mathrm{s}}\right)(1+\Delta z)=\left(1+z_{\mathrm{s}}\right)\left(1-v_{\text {Dopp }}\right)
$$

\footnotetext{
${ }^{2}$ The apparent size distance $D$ is defined as the relation between the physical size of a distant object $l$ and the (small) angle $\theta$ under which it is seen by an observer: $D=l / \theta$
}

In the case of a surrounding Minkowski metric, the redshift factors become unity and the distance parameters become additive. In this case Eq. (37) predicts a zero Doppler velocity if source, lens and observer are all moving with the same velocity, as expected. The combination of velocities is very similar to the effective transversal velocity $V$ defined by Kayser, Refsdal, and Stabell [27, Eq. (B9)] to discuss effects of microlensing. The only difference (besides the multiplication with $\Delta \theta$ ) is that their $V$ is projected into the source plane and incorporates a cosmological time dilation factor, so that

$$
v_{\text {Dopp }}=\left(1+z_{\mathrm{d}}\right) \frac{D_{\mathrm{od}}}{D_{\mathrm{ds}}} V \Delta \theta .
$$

If more than one lensed image of the source is observed, the effect of the transversal motion can be separated from the $a$ priori unknown radial velocity (including cosmological redshift) of the source, which usually contributes a much larger Doppler effect. In this case the radial velocity will have the same effect on all images while the effect of transversal motion depends on the position of the image. The latter can therefore be measured unambiguously by comparing the Doppler shifts of at least two images. This possibility makes the situation conceptually very different from the effects of radial motion on deflection angles which cannot be distinguished from a scaling of the total mass of the lens.

Unfortunately the effects are so small that it is hardly possible to detect them with current state-of-the-art instruments, see e.g., [19]. However, telescopes planned for the future, in particular radio arrays like ALMA ("Atacama Large Millimeter Array") or SKA ("Square Kilometer Array"), will provide sufficient sensitivity and spectral resolution to measure the effect for sources with sharp spectral features to facilitate a sufficiently accurate determination of redshifts.

\section{SUMMARY}

In this work we used different methods to calculate the effect of radially moving lenses on the deflection of light as well as test particles with arbitrary velocity. These calculations are valid for arbitrary lens velocities and thus generalize the results of previous publications (e.g., [12, 17, 18, 19]) dealing with the deflection of light by slowly moving lenses (FOV). At the same time we have demonstrated how neglecting the time dependence of the potential of a moving lens (which is equivalent to the scaling of the interaction time) accounts for the discrepancy between our results and those of [13, 14, 15, 16]. In agreement with [18] we find the inclusion of this time dependence essential for obtaining correct deflection angles.

Our study of the deflection of light and particles by radially moving lenses has revealed some rather surprising results. While a motion of the lens parallel to the test particle velocity increases the deflection of slowly moving 
particles, as is intuitively expected from the decreasing relative velocity and increasing interaction time, the opposite is true for the deflection of light. As a consequence there exists a critical particle velocity of $w_{\mathrm{c}}=1 / \sqrt{3}$ for which the motion of the lens does not have any effect on the deflection to first order. These results imply that the classical approach of treating light as classical particles, while providing qualitatively correct results for lenses at rest (see e.g., Soldner [7]), does not facilitate a satisfactory description for the case of radially moving lenses. This behavior seems to be related to the effects found by Carmeli 23, 24] and Blinnikov et al. [25, 26] for radial motion in the Schwarzschild metric where massive particles are accelerated when approaching the center only for $w<w_{\mathrm{c}}$ but decelerated otherwise.

As a further surprising result we find that the deflection angle of light vanishes in the limit of gravitational lenses approaching the observer with highly relativistic velocities, even though the effective mass-energy of the lens diverges in this limit. Intuitively one would have rather expected a diverging deflection angle in this scenario. In Appendix B we provide a descriptive explanation of this effect by demonstrating how gravitational lensing can be formulated in terms of light propagating in a refractive medium, both for static and moving lenses. In this picture the coordinate velocity of light is reduced in the rest frame of the lens. For the case of a relativistically moving lens, however, this reduction is effectively compensated by the motion of the rest frame of the lens with respect to the observer, so that the net reduction vanishes. The unchanged velocity of light in turn implies a vanishing deflection angle.

We have addressed the question whether the deflection of test particles or light can provide information about the internal structure of a rigidly moving, compact lens beyond its mass and total momentum. We find that this is not possible in the case of lenses that remain compact during the passage of the test particle.

In Appendix $C$ we additionally extend our discussion beyond the pure deflection of test particles and calculate the effects of a moving lens on the inertial system of an observer at rest. We find the motion of the lens to give rise to a dragging of this local inertial frame similar to the frame dragging known in the case of rotating stars or Kerr black holes.
In order to study the effects caused by transversally moving lenses we have used kinematic arguments in the framework of special relativity. These calculations predict a change of the radial momentum of the deflected test particle. In the case of light this manifests itself in a change of wavelength. In spite of their simplicity our kinematic calculations extend previous discussions (using general relativistic integrations along the geodesics) to arbitrary velocities of the lens and the test particles.

Finally, we have investigated the total Doppler shift arising from a moving lens in combination with a transversal motion of the source and the observer. The resulting effective Doppler velocity is similar to the total effective transversal velocities as commonly defined in studies of microlensing effects in cosmological lenses. We have thus been able to directly relate observable quantities with transversal velocities of source, lens, and observer. The required accuracy to apply this method to measure transversal velocities of astronomical objects, especially galaxies, is beyond the capability of current astronomical instruments but will be reached with the next generation of telescopes.

\section{Acknowledgments}

O.W. was supported by the BMBF/DLR Verbundforschung under grant 50 OR 0208. U.S. acknowledges the support of the Center for Gravitational Wave Physics funded by the National Science Foundation under Cooperative Agreement PHY-0114375. Work partially supported by NSF grant PHY-9800973 to Pennsylvania State University.

\section{APPENDIX A: THE GEODESIC EQUATIONS WITHOUT FOD APPROXIMATION}

We consider the boosted weak-field limit of the Schwarzschild metric Eq. (12). The geodesic equations are derived from the Euler-Lagrange equation (14) and the analogues for $t$ and $z$. In contrast to the analytic treatment of Sec. IIIB we do not use the FOD approximation and obtain the equations

$$
\begin{aligned}
0= & \left(2+4 \frac{1+v^{2}}{1-v^{2}} \Phi\right) \ddot{t}-8 \frac{v}{1-v^{2}} \Phi \ddot{z}-2 \frac{v\left(1+v^{2}\right)}{\left(1-v^{2}\right)^{3 / 2}} \Phi_{z^{\prime}} \dot{t}^{2}+4 \frac{1+v^{2}}{1-v^{2}} \Phi_{y^{\prime}} \dot{t} \dot{y} \\
& +4 \frac{1+v^{2}}{\left(1-v^{2}\right)^{3 / 2}} \Phi_{z^{\prime}} \dot{t} \dot{z}+2 \frac{v}{\sqrt{1-v^{2}}} \Phi_{z^{\prime}} \dot{y}^{2}-8 \frac{v}{1-v^{2}} \Phi_{y^{\prime}} \dot{y} \dot{z}-2 \frac{v\left(3-v^{2}\right)}{\left(1-v^{2}\right)^{3 / 2}} \Phi_{z^{\prime}} \dot{z}^{2}, \\
0= & (-2+4 \Phi) \ddot{y}-2 \frac{1+v^{2}}{1-v^{2}} \Phi_{y^{\prime}} \dot{t}^{2}-4 \frac{v}{\sqrt{1-v^{2}}} \Phi_{z^{\prime}} \dot{t} \dot{y}+8 \frac{v}{1-v^{2}} \Phi_{y^{\prime}} \dot{t} \dot{z} \\
& +2 \Phi_{y^{\prime}} \dot{y}^{2}+4 \frac{1}{\sqrt{1-v^{2}}} \Phi_{z^{\prime}} \dot{y} \dot{z}-2 \frac{1+v^{2}}{1-v^{2}} \Phi_{y^{\prime}} \dot{z}^{2},
\end{aligned}
$$




$$
\begin{aligned}
0= & -8 \frac{v}{1-v^{2}} \Phi \ddot{t}+\left(-2+4 \frac{1+v^{2}}{1-v^{2}} \Phi\right) \ddot{z}-2 \frac{1-3 v^{2}}{\left(1-v^{2}\right)^{3 / 2}} \Phi_{z^{\prime}} \dot{t}^{2}-8 \frac{v}{1-v^{2}} \Phi_{y^{\prime}} \dot{t} \dot{y} \\
& -4 \frac{v\left(1+v^{2}\right)}{\left(1-v^{2}\right)^{3 / 2}} \Phi_{z^{\prime}} \dot{t} \dot{z}-2 \frac{1}{\sqrt{1-v^{2}}} \Phi_{z^{\prime}} \dot{y}^{2}+4 \frac{1+v^{2}}{1-v^{2}} \Phi_{y^{\prime}} \dot{y} \dot{z}+2 \frac{1+v^{2}}{\left(1-v^{2}\right)^{3 / 2}} \Phi_{z^{\prime}} \dot{z}^{2} .
\end{aligned}
$$

In order to complete this set of equations we still need one further condition which is given by the requirement that the geodesics be null:

$$
0=g_{t t} \dot{t}^{2}+g_{y y} \dot{y}^{2}+g_{z z} \dot{z}^{2}+2 g_{t z} \dot{t} \dot{z}
$$

The potential of the lens is assumed to be that of a point mass given in the weak-field limit by

$$
\Phi\left(t^{\prime}, y^{\prime}, z^{\prime}\right)=-\frac{M}{\sqrt{y^{\prime 2}+z^{\prime 2}}}
$$

in the lens' rest frame. The resulting potential in the observer's frame is obtained from Lorentz transformation. For the derivatives that implies

$$
\begin{aligned}
& \Phi_{y}=\Phi_{y^{\prime}}, \\
& \Phi_{z}=\gamma \Phi_{z^{\prime}}, \\
& \Phi_{t}=-v \gamma \Phi_{z^{\prime}},
\end{aligned}
$$

which has already been incorporated into Eqs. A1 (A3). In order to numerically obtain a solution we need to specify boundary conditions for the null geodesic. At the point of emission of the light we demand

$$
\begin{aligned}
& t=0, \\
& z=z_{\mathrm{s}}, \\
& y=y_{\mathrm{s}}, \\
& \dot{y}=0,
\end{aligned}
$$

where $z_{\mathrm{s}}$ and $y_{\mathrm{s}}$ give the position of the source and have been set to -10000 and 150, respectively, in our calculations. At the observer's position we impose the boundary condition

$$
z=z_{\mathrm{o}}
$$

with the position of the observer being set to $z_{\mathrm{o}}=10000$ in our calculation. Note that the $y$ position of the observer depends on the deflection of the light ray and cannot be freely specified.

We solve this two-point-boundary value problem with a second order accurate relaxation scheme. For our calculations we have set the mass of the lens to $M=1$, so that all distances are given in units of the lens' mass (remember that $c=1=G$ ). These parameters lead to deflection angles much larger than those observed in astronomical lenses. We therefore expect this test of the FOD approximation to provide upper bounds on the error and the FOD approximation to be even more accurate in all scenarios of practical interest.

\section{APPENDIX B: INDEX OF REFRACTION}

In this section we will provide an alternative descriptive explanation of the counterintuitive result obtained in Secs. II B and IIC that a gravitational lens moving towards the observer at highly relativistic speed leads to a vanishing deflection angle. We have not found an intuitive explanation for this effect based on the components of the energy momentum tensor which diverge in the limit of $v \rightarrow 1$, but whose contributions must cancel to explain the vanishing total effect. Instead we describe the deflection in terms of light moving in a refractive medium.

It is a well known fact that the deflection of light in weak static gravitational fields can be described by the analogous scenario of light moving in Euclidean space filled with a refractive medium. We therefore consider a refractive medium in the rest frame of the lens. We denote the propagation speed of light in the medium by $c^{\prime}$ so that the refractive index is given by

$$
n^{\prime}=\frac{1}{c^{\prime}}=\frac{\mathrm{d} t^{\prime}}{\mathrm{d} l^{\prime}} .
$$

As before we use primed coordinates for the rest frame of the lens. Note, however, that the spatial geometry is viewed as Euclidian now, so that $\mathrm{d} l^{\prime 2}=\mathrm{d} y^{\prime 2}+\mathrm{d} z^{\prime 2}$. The gravitational effect on the light propagation is absorbed in the refractive index

$$
n^{\prime}-1=-2 \Phi
$$

which follows from the combination of Eq. (B1) and the line element (11) for the case of light (i.e., $\mathrm{d} s^{2}=0$ ). This description can be extended to the nonstatic scenario in the rest frame of the observer either by applying the Lorentz transformation (9)-111) or by directly calculating $n$ as before but now using the unprimed analogue of Eq. (B1) and the metric (12) for the moving lens. Both approaches lead to the rather simple scaling law

$$
\frac{n-1}{n^{\prime}-1}=\gamma^{2}(1-v \cos \theta)^{2}
$$

Here $\theta$ defines the direction of light propagation relative to the lens' motion as seen by the observer, i.e., $\cos \theta=$ $\mathrm{d} z / \mathrm{d} l, \sin \theta=\mathrm{d} y / \mathrm{d} l$. This result is the generalization of the FOV expression of Schneider et al. [4]. It is also valid for arbitrary directions $\theta$, even though we are still working in the FOD approximation.

We emphasize that we have obtained a description of a moving gravitational potential in terms of a refractive 
medium at rest but with an anisotropic refractive index $n$ given by Eqs. (B2) and (B3). In particular this scaling law demonstrates that the refractive index $n$ approaches unity in the limit of the gravitational lens moving towards the observer with highly relativistic velocity $(\theta=0, v \rightarrow$ 1). Translated back to the deflection of light, however, a constant refractive index $n \equiv 1$ implies a vanishing of the deflection angle.

It is now not surprising at all that the refractive index becomes unity in this limit. The coordinate velocity of light is reduced in the rest frame of the lens, but as the lens itself is moving highly relativistically, the resulting net velocity in the observer's frame is obtained from relativistic velocity addition [cf. Eq. (21)] and approaches 1. In the limit $v \rightarrow 1$ we thus have no reduction of the velocity of light in the refractive medium and consequently no deflection.

\section{APPENDIX C: FRAME DRAGGING}

The fact that the velocity of light is reduced in a moving frame of reference (cf. Appendix (B) suggests effects analogous to the "dragging of inertial frames" in the field of a rotating body with its extreme consequences close to Kerr black holes. A discussion of this effect helps in understanding the geometric effects in the field of a linearly moving mass and is therefore included in this appendix. Gabriel, Haugan, and Zhou [28] showed that the gravitomagnetic light deflection of rotating bodies can be expressed as an integral over the Lense-Thirring rotation rate which demonstrates the close relation between both effects.

To simplify the discussion we consider regions with a stationary (static in the lens frame) gravitational field in $y$ direction, i.e., $\Phi_{z}=\Phi_{t}=0$. It can be shown easily that a freely falling particle will gain a velocity component in the $z$ direction (parallel to the direction of motion of the lens) as a result of its motion in the $y$ direction. This transversal acceleration is similar to magnetic Lorentz forces and shows the gravitomagnetic effects directly. Such effects are, however, coordinate dependent and thus not easy to interpret in a general way. We therefore want to discuss effects on a body at rest with respect to the outside world, i.e., with constant $y$ and $z$ coordinates.

The tetrad of a local Minkowski coordinate system can be derived by starting at one point of the world line and transforming the direction vectors $T^{\nu}$ with the FermiWalker transport which is defined by a vanishing Fermi derivative,

$$
\frac{\mathrm{D} T^{\alpha}}{\mathrm{D} \tau}+g_{\mu \nu}\left(u^{\alpha} \frac{\mathrm{D} u^{\mu}}{\mathrm{D} \tau}-u^{\mu} \frac{\mathrm{D} u^{\alpha}}{\mathrm{D} \tau}\right) T^{\nu}=0 .
$$

Here the four-velocity of the test particle is denoted by $u^{\alpha}=\mathrm{d} x^{\alpha} / \mathrm{d} \tau$ (with $\tau$ being the proper time along the trajectory of the test particle) and the covariant deriva- tives by an uppercase D:

$$
\frac{\mathrm{D} T^{\alpha}}{\mathrm{D} \tau}=\frac{\mathrm{d} T^{\alpha}}{\mathrm{d} \tau}+\Gamma_{\mu \nu}^{\alpha} T^{\mu} u^{\nu}
$$

In order to derive the Christoffel symbols we write the Euler-Lagrange equations [e.g., Eq. (14)] for the Lagrange function in Eq. (13). On the other hand we write the equations of motion by setting the covariant derivative of the velocity vector $T^{\alpha}=u^{\alpha}$ in Eq. (C2) to zero. Even though we still work in the FOD approximation, we now do allow for arbitrary directions of test particle velocities. By comparing coefficients, we find the following nonvanishing Christoffel symbols:

$$
\begin{array}{ll}
\Gamma_{t y}^{t}=\left(1+v^{2}\right) \gamma^{2} \Phi_{y}, & \Gamma_{y z}^{t}=-2 v \gamma^{2} \Phi_{y}, \\
\Gamma_{t t}^{y}=\left(1+v^{2}\right) \gamma^{2} \Phi_{y}, & \Gamma_{y y}^{y}=-\Phi_{y}, \\
\Gamma_{z z}^{y}=\left(1+v^{2}\right) \gamma^{2} \Phi_{y}, & \Gamma_{t z}^{y}=-2 v \gamma^{2} \Phi_{y}, \\
\Gamma_{t y}^{z}=2 v \gamma^{2} \Phi_{y}, & \Gamma_{y z}^{z}=-\left(1+v^{2}\right) \gamma^{2} \Phi_{y},
\end{array}
$$

as well as those following from the symmetry properties. The four-velocity $u^{\alpha}$ of a particle at rest is needed only to zeroth order of the deflection (i.e., potential) and is given by $u^{t}=1, u^{z}=u^{y}=0$ in unprimed coordinates. For the covariant derivative of $u^{\alpha}$ for the particle at rest, we obtain in FOD approximation

$$
\frac{\mathrm{D} u^{y}}{\mathrm{D} \tau}=\left(1+v^{2}\right) \gamma^{2} \Phi_{y}
$$

while all other components are vanishing. This covariant derivative for the particle at rest is equivalent to the force needed to support the particle and prevent it from falling towards the lens.

In the next step we apply Eqs. (C1) and (C2) to derive the transformation of the components of an arbitrary vector $T^{\alpha}$. For the metric $g_{\mu \nu}$, the zeroth order approximation (i.e., the Minkowski metric) is sufficient in this case. We arrive at the final equations for the FermiWalker transport:

$$
\begin{aligned}
\frac{\mathrm{d} T^{\alpha}}{\mathrm{d} \tau} & =M_{\beta}^{\alpha} T^{\beta} \\
\left(M^{\alpha}{ }_{\beta}\right) & =2 v \gamma^{2} \Phi_{y}\left(\begin{array}{ccc}
0 & 0 & 0 \\
0 & 0 & 1 \\
0 & -1 & 0
\end{array}\right)
\end{aligned}
$$

The ordering of indices is $t, y, z$, the row index is $\alpha$, and the column index $\beta$. We note that the velocity vector $u^{\alpha}$ itself remains constant as required. Furthermore, we see that the $y$ and $z$ coordinates evolve in a way which is equivalent to a rotation. Modulo an arbitrary time offset, the fundamental solution of this part is

$$
T^{y}=\cos (\omega \tau), \quad T^{z}=\sin (\omega \tau)
$$

with an angular velocity of $\omega=-2 v \gamma^{2} \Phi_{y}$. As a consequence a gyroscope (or any other device capable of 
sustaining a locally nonrotating reference system) will precess relative to the outside world with this angular velocity. This precession can be observed from great distance and represents a coordinate independent measure of the frame dragging caused by the linear motion of the lens. ${ }^{3}$

Without presenting the calculations we note that the precession of a gyroscope could be obtained more directly by solving the equation of motion for the different parts of the rotating mass. The mass elements approaching the lens will be dragged with the lens' motion while elements moving parallel to the lens will feel a reduced gravitational attraction. It is easy to show that this leads ex-

3 The assumption of constant spatial coordinates for the test particle is not coordinate dependent because it can be defined in the way that the test particle appears to be at rest when observed by actly to the precession which we derived in a more formal but general way.

The effect is analogous to Lense-Thirring precession in the field of rotating bodies, especially in the field of a Kerr black hole. There is, however, one important difference. In our case, all gravitomagnetic terms of the metric vanish in the rest frame of the lens. The precession due to the frame dragging does of course not disappear when viewed in this system. It would instead be interpreted as an incarnation of geodesic precession of a moving particle in a static gravitational field. In the case of the Kerr metric, such a transformation is possible locally only as has been shown by Ashby and Shahid-Saless [29].

a distant observer whose coordinates are fixed by the background Minkowski metric.
[1] R. D. Blandford and R. Narayan, Annu. Rev. Astron. Astrophys. 30, 311 (1992).

[2] S. Refsdal and J. Surdej, Rep. Prog. Phys. 57, 117 (1994).

[3] R. Narayan and M. Bartelmann, in Formation of Structure in the Universe, Proceedings of the 1995 Jerusalem Winter School, edited by A. Dekel and J. P. Ostriker (Cambridge University Press, 1999), p. 360, also e-print astro-ph/9606001.

[4] P. Schneider, J. Ehlers, and E. E. Falco, Gravitational Lenses (Springer-Verlag, A\&A Library, 1992).

[5] F. Dyson, A. Eddington, and C. Davidson, Philos. Trans. R. Soc. London A220, 291 (1920).

[6] C. M. Will, Am. J. Phys. 56, 413 (1988).

[7] J. Soldner, in Berliner Astronomisches Jahrbuch 1804 (Akademie-Verlag, Berlin, 1801), p. 161.

[8] A. Einstein, Ann. Phys. (Leipzig) 35, 898 (1911).

[9] A. Einstein, in Sitzungsberichte der Preußischen Akademie der Wissenschaften, erster Halbband (1915), p. 831.

[10] D. S. Robertson, W. E. Carter, and W. H. Dillinger, Nature (London) 349, 768 (1991).

[11] D. E. Lebach et al., Phys. Rev. Lett. 75, 1439 (1995).

[12] T. Pyne and M. Birkinshaw, Astrophys. J. 415, 459 (1993).

[13] S. Capozziello, G. Lambiase, G. Papini, and G. Scarpetta, Phys. Lett. A 254, 11 (1999), also e-print astroph/9902373.

[14] S. Capozziello and V. Re, Phys. Lett. A 290, 115 (2001), also e-print astro-ph/0109547.

[15] M. Sereno, Phys. Lett. A 305, 7 (2002), also e-print astro- $\mathrm{ph} / 0209148$.

[16] M. Sereno and V. F. Cardone, Astron. Astrophys. 396 , 393 (2002), also e-print astro-ph/0209297.

[17] S. Frittelli, T. P. Kling, and E. T. Newman, Phys. Rev. D 65, 123007 (2002), also e-print gr-qc/0205014.

[18] S. Frittelli, Mon. Not. R. Astron. Soc. 340, 457 (2003), also e-print astro-ph/0212207.

[19] S. Molnar and M. Birkinshaw, Astrophys. J. 586, 731 (2003), also e-print astro-ph/0211227.

[20] S. M. Kopeikin and G. Schäfer, Phys. Rev. D 60, 124002 (1999).

[21] A. A. de Laix and T. Vachaspati, Phys. Rev. D 54, 4780 (1996).

[22] J. P. Uzan and F. Bernardeau, Phys. Rev. D 63, 23004 (2001).

[23] M. Carmeli, Lett. Nuovo Cimento Soc. Ital. Fis. 3, 379 (1972).

[24] M. Carmeli, Classical Fields: General Relativity and Gauge Theory (Wiley, New York, 1982).

[25] S. I. Blinnikov, L. B. Okun, and M. I. Vysotsky (2001), e-print gr-qc/0111103.

[26] S. I. Blinnikov, L. B. Okun, and M. I. Vysotsky (2003), e-print gr-qc/0310020.

[27] R. Kayser, S. Refsdal, and R. Stabell, Astron. Astrophys. 166, 36 (1986).

[28] M. D. Gabriel, M. P. Haugan, and Z. Zhou, Phys. Rev. D 37, 2699 (1988).

[29] N. Ashby and B. Shahid-Saless, Phys. Rev. D 42, 1118 (1990). 